Research Article

Yavuz Can*, Önder Yaz, and Dietmar Fey

\title{
Disjoint Sum of Products by Orthogonalizing Difference-Building $\ominus$
}

https://doi.org/10.1515/eng-2020-0067

Received May 21, 2019; accepted May 07, 2020

\begin{abstract}
The orthogonalization of Boolean functions in disjunctive form, that means a Boolean function formed by sum of products, is a classical problem in the Boolean algebra. In this work, the novel methodology $O R T H[\ominus]$ of orthogonalization which is an universally valid formula based on the combination technique »orthogonalizing difference-building $\ominus$ « is presented. Therefore, the technique $\ominus$ is used to transform Sum of Products into disjoint Sum of Products. The scope of orthogonalization will be solved by a novel formula in a mathematically easier way. By a further procedure step of sorting product terms, a minimized disjoint Sum of Products can be reached. Compared to other methods or heuristics $O R T H[\ominus]$ provides a faster computation time.
\end{abstract}

Keywords: Sum of Products, disjoint Sum of Products, Minimization, Orthogonalization, Disjunctive Form

\section{Introduction and Preliminaries}

A Boolean function of $n$ variables is defined as the mapping $f(\underline{x}):\{0,1\}^{n} \rightarrow\{0,1\}$. Four normal forms of Boolean functions exist, the disjunctive normal form (DNF), conjunctive normal form (CNF), antivalence normal form (ANF) and equivalence normal form (ENF), which consist of either product terms $p_{k}(\underline{x}):=\bigwedge_{i=1}^{n} x_{i}=$ $x_{1} \wedge . . \wedge x_{n}$ or sum of terms $s_{k}(\underline{x}):=\bigvee_{i=1}^{n} x_{i}=x_{1} \vee . . \vee x_{n}$ (with $n \geq 1$ as the number of the variables; dimension) in which variables are either negated $\bar{x}_{i}$ or not-negated

\footnotetext{
*Corresponding Author: Yavuz Can: Chair of Computer Science Friedrich-Alexander-University Erlangen-Nuremberg, GERMANY, E-mail: yavuz.can@fau.de

Önder Yaz: Chair of Computer Science, Friedrich-AlexanderUniversity Erlangen-Nuremberg, Germany;

Email: oender.yaz@fau.de

Dietmar Fey: Chair of Computer Science, Friedrich-AlexanderUniversity Erlangen-Nuremberg, Germany;

Email: dietmar.fey@fau.de
}

๖ Open Access. @ 2020 Y. Can et al., published by De Gruyter. $x_{i}[1,2]$. The normal form is the canonical representation of the Boolean function. That means that all given variables are included in a product term or sum of term respectively. The reduced form, i.e. non-canonical representation of terms, are called disjunctive, conjunctive, antivalence and equivalence forms $\mathrm{DF}, \mathrm{CF}, \mathrm{AF}$ and $\mathrm{EF}$. The disjunctive form is also considered as the Sum of Products (SOP) and notes as

$$
S O P(\underline{x}):=\bigvee_{k=1}^{N} p_{k}(\underline{x}) \text { with } \quad p_{k}(\underline{x}):=\bigwedge_{i \in A} x_{i} .
$$

$A$ is the index set of the running index $i$. The AF is a special form of Exclusive-Or Sum of Products (ESOP) and is defined as

$$
\operatorname{ESOP}(\underline{x}):=\bigoplus_{k=1}^{N} p_{k}(\underline{x}),
$$

with $N>1$ as the upper bound of the number of the product terms [?].

The orthogonality of a Boolean functions is a special attribute. A function is orthogonal if their terms have the characteristic of being disjoint in pairs in at least one variable. Thus, the following applies for the disjoint Sum of Products (dSOP):

$$
\operatorname{dSOP}(\underline{x}):=\bigvee_{k=1}^{N} p_{k}(\underline{x}) \quad \text { whereby } \quad p_{i}(\underline{x}) \wedge p_{j}(\underline{x})=0 .
$$

An orthogonal representation of a SOP, that means dSOP, is characterized by product terms which are disjoint to one another in pairs [3, 4]. Consequently, the intersection of these product terms results in 0 . The orthogonal representation of a DF - disjoint Sum of Products - is equal to the orthogonal form of an AF - the disjoint Exclusive-Or Sum of Products. In this case, it applies $d S O P(\underline{x})=d E S O P(\underline{x})$ [3-5]. That means, the dSOP is equivalent to dESOP consisting of the same product terms and differ only in the logical connectivity between the product terms. This relationship can be explained well with the following definition out of [6], if both product terms $p_{i}(\underline{x})$ and $p_{j}(\underline{x})$ are disjoint to each other. A SOP of two product terms can be transformed into an ESOP by:

$$
p_{i}(\underline{x}) \vee p_{j}(\underline{x})=p_{i}(\underline{x}) \oplus p_{j}(\underline{x}) \oplus(\underbrace{p_{i}(\underline{x}) \wedge p_{j}(\underline{x})}_{=0}) .
$$

This work is licensed under the Creative Commons Attribution 4.0 
In the special case, that both products terms are disjoint, building their conjunction results to 0 . As $x_{i} \oplus 0=x_{i}$ is, following relation follows from the Eq. (4):

$$
p_{i}(\underline{x}) \vee p_{j}(\underline{x})=p_{i}(\underline{x}) \oplus p_{j}(\underline{x}) .
$$

In this case, the left side is equal to the right side which means that a dSOP is equivalent to a dESOP. It applies $d S O P(\underline{x})=d E S O P(\underline{x})$.
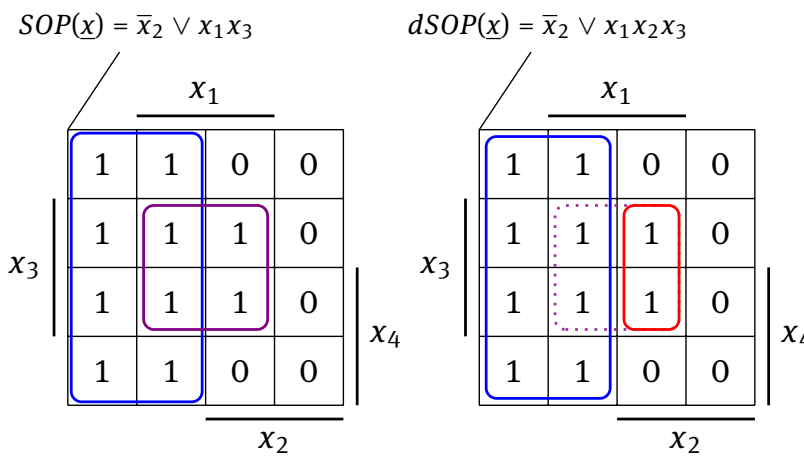

Figure 1: Difference between SOP and dSOP in a K-map

In a K-map a dSOP is characterized by nonoverlapping cubes (Figure 1). Special calculations can be easier solved in another form. It simplifies the handling of further calculations in applications of electrical engineering, e.g. calculation of suitable test patterns for combinational circuits for verifying feasible logical faults, which can mathematically be determined by Boolean Differential Calculus (BDC) [1, 7]. That means, the orthogonalization of a SOP facilitates the transformation into an equivalent dESOP $[1,3,8]$ and this characteristic simplifies the handling of BDC especially in Ternary-Vector-List (TVL) arithmetic [3,9-11]. Due to the restricted number of variables the terms of SOP are not priory disjoint. However, the disjoint form can be calculated by using a novel Boolean formula based on the novel combining technique of »orthogonalizing difference-building $\ominus \ll$.

\section{Method of Orthogonalization}

\subsection{Orthogonalizing Difference-Building $\ominus$}

Orthogonalizing difference-building $\ominus$ is the composition of two calculation steps - the usual difference-building out of the set theory and the subsequent orthogonalization, as shown in Figure 2. The result of $\ominus$ is orthogonal in contrast to the result out of the method differencebuilding. Both results are different in their representations

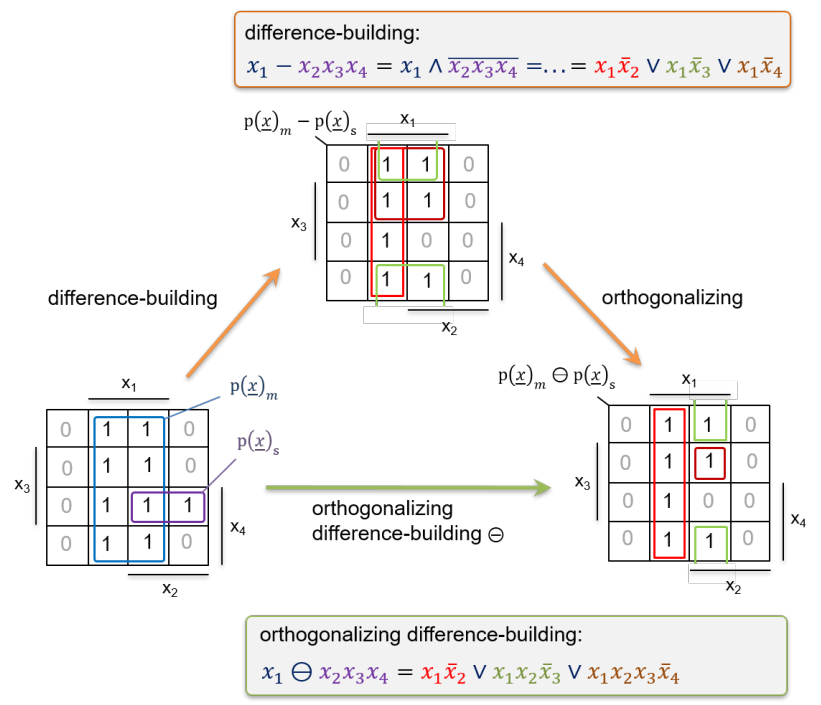

Figure 2: $\ominus$ : Two procedures in one step

but homogenous in their covering of 1s. They only differ in their form of coverage, whereas the method $\ominus$ constitutes the solution in already orthogonal form. This method $\ominus$ is generally valid and equivalent to the usual method of difference-building [3]. The orthogonalizing differencebuilding $\ominus$ corresponds to the removal of the intersection which is formed between the minuend product term $p_{m}(\underline{x})$ and the subtrahend product term $p_{s}(x)$ from the minuend product term $p_{m}(\underline{x})$, which means $p_{m}(\underline{x})-\left(p_{m}(\underline{x}) \wedge p_{s}(\underline{x})\right)$. The result consists of several product terms which are pairwise disjoint to each other. The Equation (6) applies with $n, n^{\prime} \in \mathbb{N}$ as the dimension of $p_{m}(\underline{x})$ and $p_{s}(\underline{x})$. In this case, the formula $\left(\bigvee_{i=1}^{n_{j}} \bar{x}_{i}=\bar{x}_{1_{j}} \vee x_{1_{j}} \bar{x}_{2_{j}} \vee . . \vee x_{1_{j}} x_{2_{j}} \cdot . . \bar{x}_{n_{j}}\right)$ from [4] is used to describe the orthogonalizing difference-building in a mathematically easier way. The method of orthogonalizing difference-building $\ominus$ is demonstrated by the following Example 1.

Example 1: A subtrahend $p_{s}(\underline{x})=x_{2} x_{3} x_{4}$ is subtracted from a minuend $p_{m}(\underline{x})=x_{1}$ and it appears a result, which consist of pairwise disjoint product terms.

$$
x_{1} \ominus x_{2} x_{3} x_{4}=x_{1} \bar{x}_{2} \vee x_{1} x_{2} \bar{x}_{3} \vee x_{1} x_{2} x_{3} \bar{x}_{4}
$$

The explanation of Eq. (6) is given by the following points:

- The first literal of the subtrahend, here $x_{2}$, is taken complementary and build the intersection with the minuend, here $x_{1}$. Consequently, the first term of the difference is $x_{1} \bar{x}_{2}$.

- Then the second literal, here $x_{3}$, is taken complementary and the intersection with the minuend and the first literal $x_{2}$ of the subtrahend is built. Therefore, the second term is $x_{1} x_{2} \bar{x}_{3}$. 


$$
p_{m}\left(\underline{x} \ominus p_{s}(\underline{x})=\bigwedge_{m=1}^{n} x_{m} \ominus \bigwedge_{s=1}^{n^{\prime}} x_{s}:=\bigwedge_{m=1}^{n} x_{m} \wedge \bigvee_{s=1}^{n_{j}^{\prime}} \bar{x}_{s_{j}}=\left(x_{1} \cdot \ldots \cdot x_{n}\right)_{m} \wedge\left(\bar{x}_{1_{j}} \vee \ldots \vee x_{1_{j}} \cdot \ldots \cdot x_{(n-1)_{j}} \bar{x}_{n_{j}^{\prime}}\right)_{s}\right.
$$

- Following the next literal, here $x_{4}$, is taken complementary and the intersection with the minuend and the first literal $x_{2}$ and second literal $x_{3}$ of the subtrahend is built. Thus, the third term of the difference is $x_{1} x_{2} x_{3} \bar{x}_{4}$.

- This process is continued until all literals of the subtrahend are singly complemented and linked by building the intersection with the minuend in a separate term.

$n_{\text {orth }}$ as the number of product terms in the orthogonal result corresponds to the number of literals presented in the subtrahend $p_{s}(\underline{x})$ and are not presented in the minuend $p_{m}(\underline{x})$ at the same time. Following rules must be followed to get correct results for the application of $\ominus$ :

1. If the subtrahend is already orthogonal to the minuend $\left(p_{s}(\underline{x}) \perp p_{m}(\underline{x})\right)$ the result corresponds to the minuend:

$$
p_{m}(\underline{x}) \ominus p_{s}(\underline{x})=\left.p_{m}(\underline{x})\right|_{p_{s}(\underline{x}) \perp p_{m}(\underline{x})}
$$

2. The difference between 0 and the subtrahend is the subtrahend itself:

$$
0 \ominus p_{s}(\underline{x})=p_{s}(\underline{x})
$$

3. The result between 1 and subtrahend is the complement of the subtrahend which results in a dSOP:

$$
1 \ominus p_{s}(\underline{x})=\left.d S O P(\underline{x})\right|_{p_{s}(\underline{x})}
$$

4. Thereby, the subset symbol $\subseteq$ of the set theory is transferred to switching algebra. The result between subtrahend and minuend is empty if the subtrahend is already completely contained in the minuend. If the subtrahend is the subset of the minuend $\left(p_{s}(\underline{x}) \subseteq p_{m}(\underline{x})\right)$, the result is 0 :

$$
p_{m}(\underline{x}) \ominus p_{s}(\underline{x})=\left.0\right|_{p_{s}(\underline{x}) \subseteq p_{m}(\underline{x})}
$$

\subsection{Orthogonalization of SOP}

\subsubsection{Mathematical Methodology}

The orthogonalization of every $S O P(\underline{x})$ consisting of at least two product terms $(N>1)$ can be performed by Eq. (11) which bases on the Eq. (6), which is based on the combination technique of $\ominus$ [3]. The order of the calculation is important. That means, the first two product terms must be calculated and then the third product term must be calculated with the result of the two product terms before, and so on. The result of $d S O P(\underline{x})$ can diversify depending on the starting product term. As a SOP has the characteristic of being commutative, the order of their product terms can be changed for getting results with fewer number of disjoint product terms called as $N_{\text {orth }}$. To obtain better result is often reached by ordering the product terms from higher number of variables to fewer number of variables. Following Example 2 gives an overview about the procedure orthogonalizing by Eq. (11) and afterwards the Example 3 with an additional process of sorting.

Example 2: Function $S O P_{1}(\underline{x})=\bar{x}_{3} \vee x_{1} x_{2} \vee x_{1} x_{3}$ has to be orthogonalized by Eq. (11).

$$
\begin{aligned}
d S O P_{1}(\underline{x}) & =\left(\bar{x}_{3} \ominus x_{1} x_{2} \ominus x_{1} x_{3}\right) \vee\left(x_{1} x_{2} \ominus x_{1} x_{3}\right) \vee x_{1} x_{3} \\
& =(\underbrace{\left(\bar{x}_{1} \bar{x}_{3} \vee \bar{x}_{1} \bar{x}_{2} \bar{x}_{3}\right) \ominus x_{1} x_{3}}_{\text {Eq. }(7)}) \vee x_{1} x_{2} \bar{x}_{3} \vee x_{1} x_{3} \\
& =\bar{x}_{2} \bar{x}_{3} \vee \bar{x}_{1} x_{2} \bar{x}_{3} \vee x_{1} x_{2} \bar{x}_{3} \vee x_{1} x_{3}
\end{aligned}
$$

Function $d S O P_{1}(\underline{x})$ consists of four disjoint product terms $\left(N_{\text {orth }}=4\right)$ and is the orthogonalized form of $S O P_{1}(\underline{x})$. Both are equivalent. They only differ in their form of coverage which is illustrated in the K-maps as shown in Figure 3.

Example 3: Now, the sorted function ${ }^{\text {sort }} d S O P_{1}(\underline{x})=$ $x_{1} x_{2} \vee x_{1} x_{3} \vee \bar{x}_{3}$ of Example 2 has to be orthogonalized by Eq. (11).

$$
\begin{aligned}
{ }^{\text {sort }} \operatorname{dSOP}_{1}(\underline{x}) & =\left(x_{1} x_{2} \ominus x_{1} x_{3} \ominus \bar{x}_{3}\right) \vee(\underbrace{x_{1} x_{3} \ominus \bar{x}_{3}}_{\text {Eq. }(7)}) \vee \bar{x}_{3} \\
& =(\underbrace{x_{1} x_{2} \bar{x}_{3} \ominus \bar{x}_{3}}_{=0 \text { Eq. }}) \vee x_{1} x_{3} \vee \bar{x}_{3}= \\
& =x_{1} x_{3} \vee \bar{x}_{3}
\end{aligned}
$$

Function ${ }^{\text {sort }} d S O P_{1}(\underline{x})$ is another equivalent orthogonal form of $S_{S P}(x)$ which consists of two disjoint product terms $\left(N_{\text {orth }}=2\right)$ that is also illustrated in third K-map (Figure 3). The coverage of $1 \mathrm{~s}$ is done by two cubes. By sorting, a minimized dSOP can be reached. The comparison of the three functions shows their equivalence. They are homogenous and only differ in their form of superimposition. 


$$
d S O P(\underline{x}):=\bigvee_{k=0}^{N-1}\left(\bigoplus_{i=k+1}^{N} p_{i}(\underline{x})\right)=\left(p_{1}(\underline{x}) \ominus \ldots \ominus p_{N}(\underline{x})\right) \vee\left(p_{2}(\underline{x}) \ominus \ldots \ominus p_{N}(\underline{x})\right) \vee \ldots \vee\left(p_{N-1}(\underline{x}) \ominus p_{N}(\underline{x})\right) \vee p_{N}(\underline{x})
$$
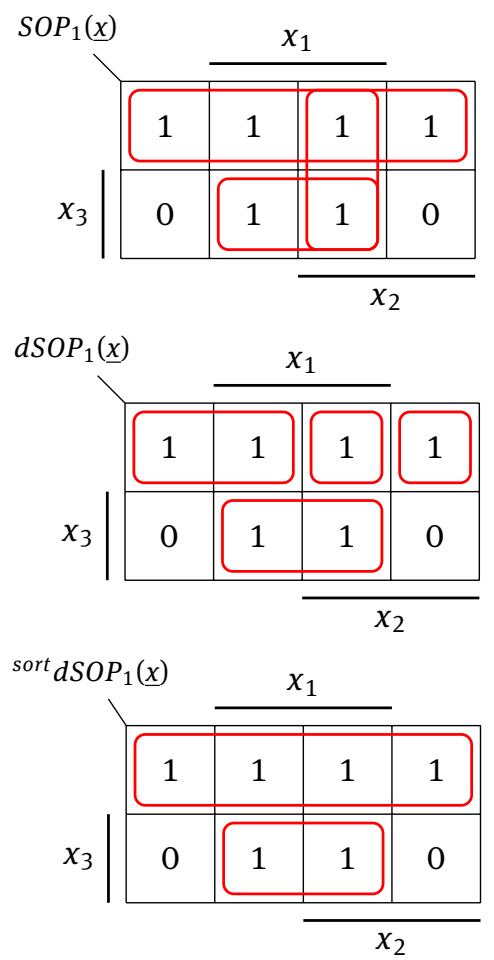

Figure 3: Comparison of $S O P_{1}(\underline{x}), d S O P_{1}(\underline{x})$ and ${ }^{\text {sort }} d S O P_{1}(\underline{x})$

\subsubsection{Algorithm}

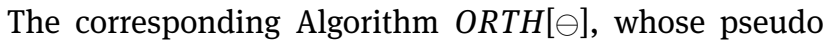
code is shon in the Table 1 outlines the computational procedure of orthogonalization of a SOP according to the formula in Eq. (11). To obtain dSOP with fewer number of product terms, the sub-functions $a b s o r b()$ and sort () are additionally used. $a b s o r b()$ is a function which reduces the number of product terms of the SOP, which serves as the input of the algorithm. The reduction is achieved by by absorption of smaller product terms, which consists of higher number of variables, by larger product terms, which consists of lower number of variables if those are already covered by the larger ones (following example):

$$
\ldots \vee x_{1} x_{2} x_{3} \vee x_{1} \bar{x}_{2} \bar{x}_{3} \vee x_{1} \vee \ldots \stackrel{\text { absorb } 0}{\longrightarrow} \ldots \vee x_{1} \vee \ldots
$$

The product term $x_{1}$ is absorbing the other two product term. Additionally, absorb() reduces duplicated product terms to a single term which is demonstrated with the following example:

$\ldots \vee x_{2} \bar{x}_{3} \vee x_{3} \vee x_{2} \bar{x}_{3} \vee \ldots \stackrel{a b s o r b()}{\longrightarrow} \ldots \vee x_{2} \bar{x}_{3} \vee x_{3} \vee \ldots$

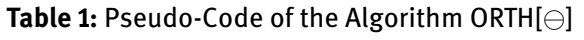

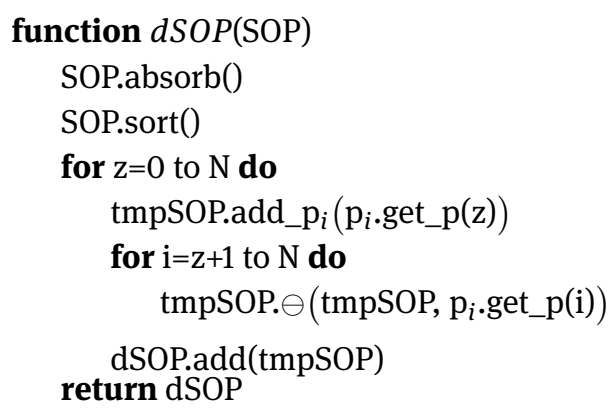

Consequently, by using absorb() the number of product terms, that have to be treated decreases. With the optionally function sort () follows the resorting of the product terms from smaller product terms to larger product terms. After proceeding these two sub-functions $a b s o r b()$ and $\operatorname{sort}()$ the process of orthogonalization $O R T H[\ominus]$ according to the method $\ominus$ is performed.

\section{Comparison and Measurement}

\section{1 $N_{\text {orth }}$ before and after Sorting}

However, to make a statement about the optimized form, the optimum minimization would have to be defined, which has not yet been clarified. Table 2 illustrates the percentage of reduced terms by the use of subsequent procedure of sorting. The procedure of sorting brings an advantage for gaining minimized dSOP. Firstly, a list of ten non-orthogonal functions in respect to $N=\{5,10,15\}$ and dimension $x_{n}=\{5,6, \ldots, 50\}$ were created. Consequently, per each $N$ has produced 50 different nonorthogonal SOPs. Subsequently, each SOP was orthogonalized according to the method $\ominus$ before and after sorting. The resulting number of product terms $N_{\text {orth }}$ in dSOP and ${ }^{\text {sort }} N_{\text {orth }}$ in ${ }^{\text {sort }} \mathrm{dSOP}$ in respect to $N$ and $x_{n}$ were determined (Figure 4) and compared. The number of product terms of a sorted SOP is fewer then the unsorted SOP. It follows ${ }^{\text {sort }} N_{\text {orth }}\left(N, x_{n}\right)<N_{\text {orth }}\left(N, x_{n}\right)$. An average value of these values was calculated for each dimension $x_{n}$. Thereby, the results of the quotients of the average number of disjoint product terms were obtained. Furthermore, 


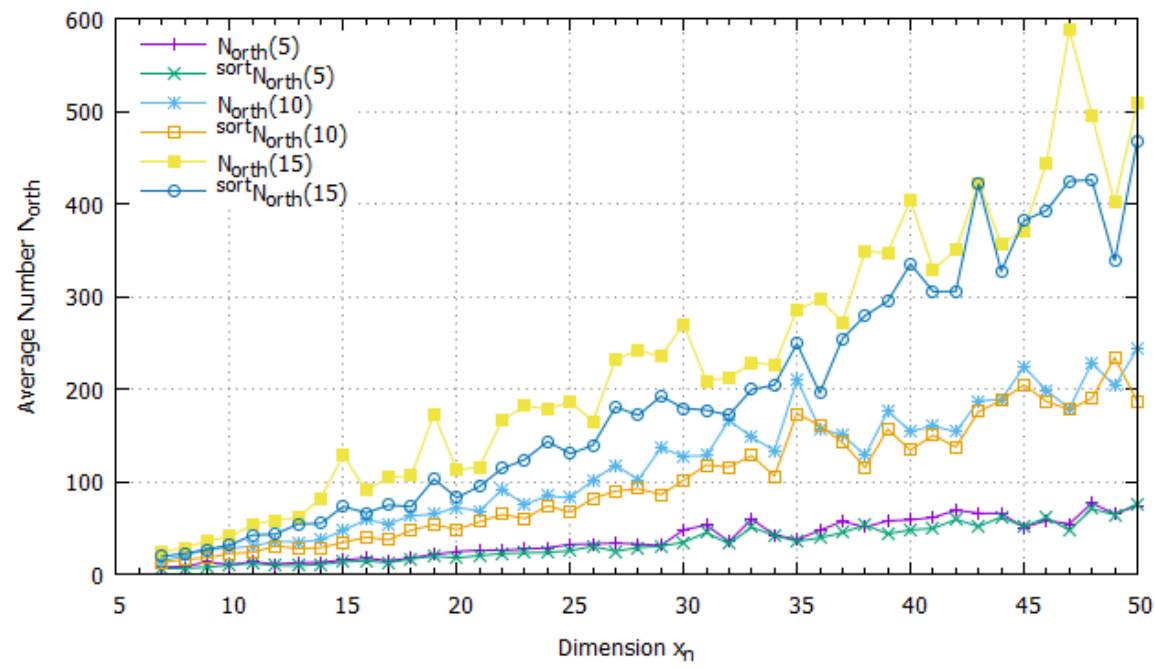

Figure 4: Average number of $N_{\text {orth }}$ and ${ }^{\text {sort }} N_{\text {orth }}$

Table 2: Percentage Value of $\frac{N_{\text {orth }}}{\text { sort } N_{\text {orth }}}$

\begin{tabular}{lccc}
\hline \multicolumn{4}{c}{ Percentage Value [\%] } \\
\hline$x_{n}$ & $\frac{N_{\text {orth }}}{\text { sort }}(5)$ & $\frac{N_{\text {orth }}}{\text { sort }}(10)$ & $\frac{N_{\text {orth }}}{\text { sort }}(15)$ \\
\hline 5 & 0.0 & 8.6 & - \\
6 & 23.6 & 29.0 & - \\
7 & 28.8 & 11.8 & 24.6 \\
8 & 30.2 & 27.0 & 28.7 \\
9 & 73.3 & 52.0 & 32.8 \\
10 & 6.1 & 24.6 & 29.6 \\
$\vdots$ & $\vdots$ & $\vdots$ & $\vdots$ \\
28 & 13.6 & 10.5 & 40.9 \\
29 & 2.7 & 58.6 & 22.3 \\
30 & 38.6 & 25.8 & 50.6 \\
31 & 17.8 & 8.5 & 18.0 \\
$\vdots$ & $\vdots$ & $\vdots$ & $\vdots$ \\
45 & -1.6 & 9.4 & -2.8 \\
46 & -5.4 & 6.5 & 13.1 \\
47 & 14.8 & 0.6 & 38.4 \\
48 & 8.8 & 20.2 & 16.4 \\
49 & 1.2 & -13.1 & 18.6 \\
50 & -2.5 & 30.6 & 8.8 \\
\hline$\varnothing$ & 17.3 & 21.7 & $\mathbf{2 7 . 6}$ \\
\hline
\end{tabular}

quotients of $N_{\text {orth }}$ and ${ }^{\text {sort }} N_{\text {orth }}$ in percentage value were gained. The minus signifies that in that case the number of $N_{\text {orth }}$ is fewer. Finally, a total average percentage value per each $N$ was determined out of these average values (Table 2). Three in their number. Consequently, an additional procedure of sorting leads to a dSOP with fewer number of product terms. Minimalization of approximately $17 \%$ till $28 \%$ is obtained in comparison to a dSOP which has not been sorted before.

\subsection{Comparison in Number of Terms $\boldsymbol{N}_{\text {orth }}$}

The number of the disjoint product terms $N_{\text {orth }}$ in a dSOP produced by $O R T H[\ominus]$ is analyzed in comparison to the heuristic ORTH[DSOP] in [5], the method $O R T H[m 1]$ in [12] and a varied form of ORTH[DSOP] as ORTH[DSOP2]. In the varied form ORTH[DSOP2] the minimization function "espresso.exe" was replaced by $a b s o r b()$. The comparisions in respect to $N=$ $\{2,5,10,15,20,25\}$ and $x_{n}=\{1,2, \ldots, 50\}$ are shown in Figure 5 . The corresponding average values $\overline{N_{\text {orth }}}$ and the ratios to the method $O R T H[\ominus]$ are given in Table 3. The average value $N_{\text {orth }}$ for each $N$ are formed of 50 calculated tasks for each dimension $x_{n}$. Out of these average values of $N_{\text {orth }}$ regarding to a method an average value $\overline{N_{\text {orth }}}$ is built. The corresponding charts, as shown in the Figures 5a) - f), illustrate that the method $O R T H[\ominus]$ offers results with fewer number of terms $N_{\text {orth }}$ in respect to growing $x_{n}$ and $N$ in comparison to the methods $O R T H[m 1]$ and $O R T H[D S O P 2]$. The number of terms are 1.67 times fewer by $O R T H[\ominus]$ in comparison to ORTH[m1]. Against this, the heuristic ORTH[DSOP] provides approximately $50 \%$ fewer number of terms $N_{\text {orth }}$ in contrast to the method $O R T H[\ominus]$. Therefore the relationship $N_{\text {orth }}^{O R T H[D S O P]}<N_{\text {orth }}^{O R T H[\ominus]}<N_{\text {orth }}^{O R T H[m 1]}$ can be deduced from this. Probably, this benefit is given by the use of "espresso.exe" , which is a program used as heuris- 


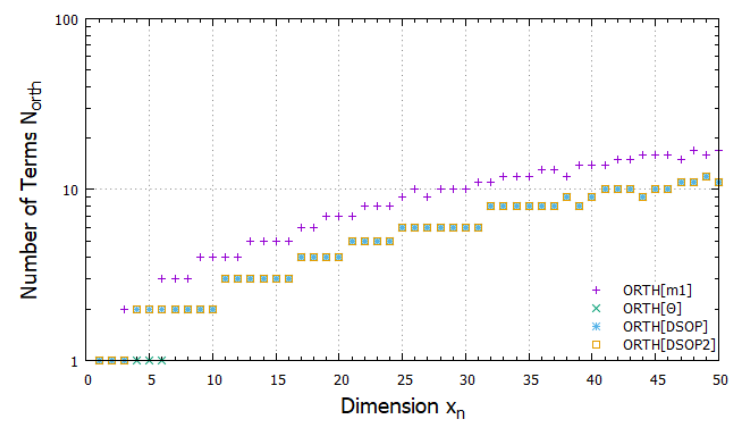

(a) SOPs with 2 product terms

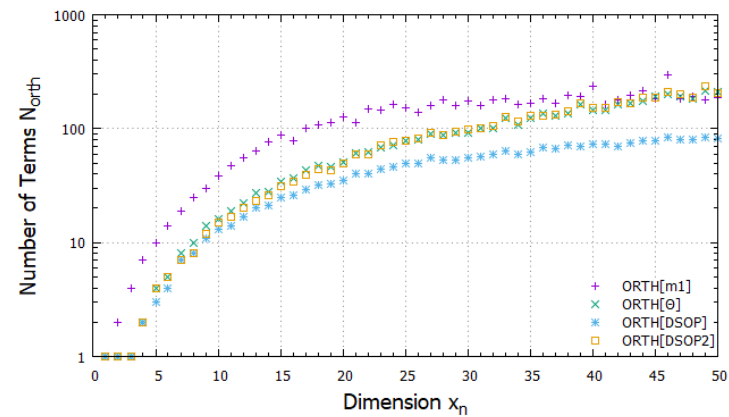

(c) SOPs with 10 product terms

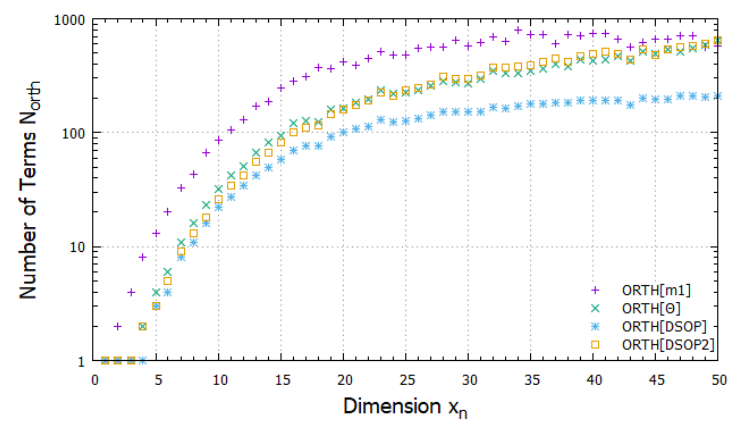

(e) SOPs with 20 product terms

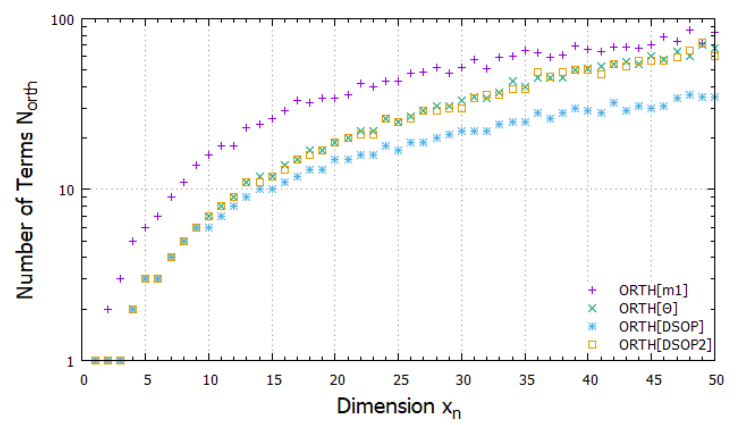

(b) SOPs with 5 product terms

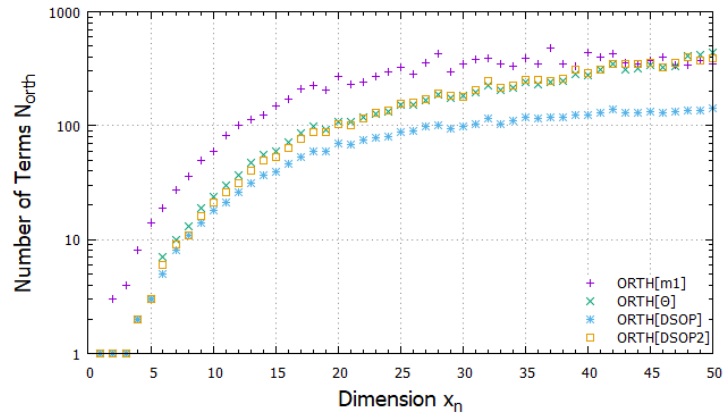

(d) SOPs with 15 product terms

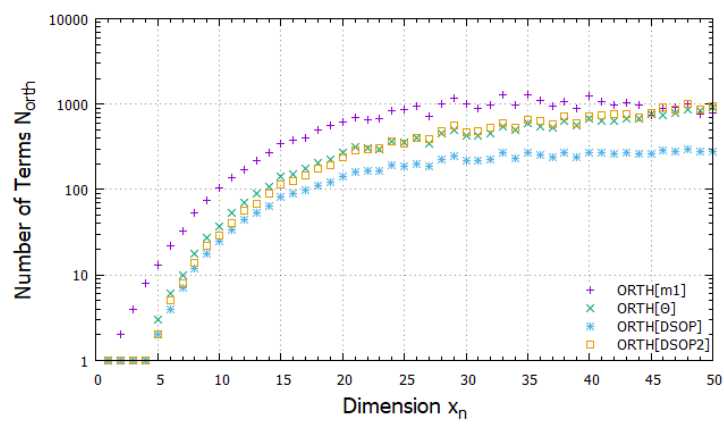

(f) SOPs with 25 product terms

Figure 5: Average number of the $N_{\text {orth }}$ in the dSOP

Table 3: Average of the number of terms $N_{\text {orth }}$ and relation to $O R T H[\ominus]$

\begin{tabular}{|c|c|c|c|c|c|c|c|}
\hline$x_{n}$ & 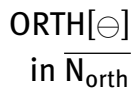 & $\begin{array}{r}\text { ORTH[DSOP] } \\
\text { in } \overline{N_{\text {orth }}}\end{array}$ & $\begin{array}{r}\text { ORTH[DSOP2] } \\
\text { in } \overline{N_{\text {orth }}}\end{array}$ & $\begin{array}{l}\mathrm{ORTH}[\mathrm{m} 1] \\
\text { in } \overline{\mathrm{N}_{\text {orth }}}\end{array}$ & 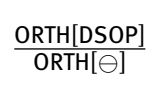 & $\frac{\text { ORTH[DSOP2] }}{\text { ORTH }[\ominus]}$ & $\frac{\mathrm{ORTH}[\mathrm{m} 1]}{\mathrm{ORTH}[\ominus]}$ \\
\hline 2 & 6,22 & 6,30 & 6,30 & 9,45 & 1,01 & 1,01 & 1,52 \\
\hline 5 & 29,52 & 18,57 & 29,11 & 43,22 & 0,63 & 0,99 & 1,46 \\
\hline 10 & 86,72 & 44,96 & 88,32 & 127,35 & 0,52 & 1,02 & 1,47 \\
\hline 15 & 164,05 & 77,82 & 165,90 & 249,31 & 0,47 & 1,01 & 1,52 \\
\hline 20 & 248,18 & 115,97 & 258,58 & 431,67 & 0,47 & 1,04 & 1,74 \\
\hline 25 & 369,09 & 162,79 & 393,47 & 650,57 & 0,44 & 1,07 & 1,76 \\
\hline$\varnothing$ & 150,63 & 71,07 & 156,95 & 251,93 & 0,47 & 1,04 & 1,67 \\
\hline
\end{tabular}




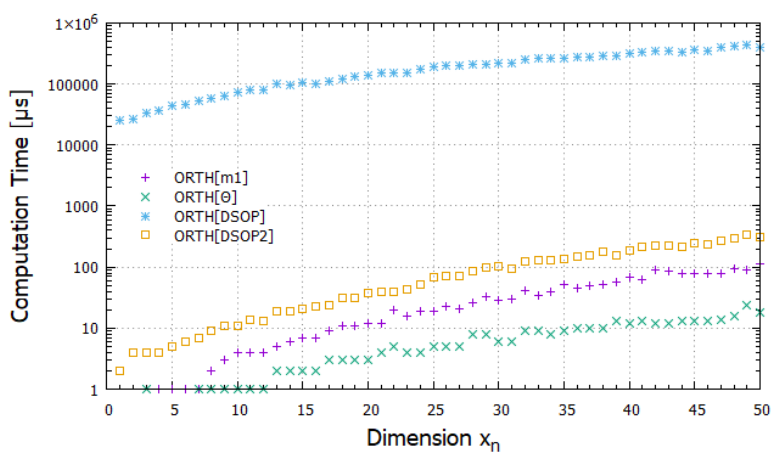

(a) SOPs with 2 product terms

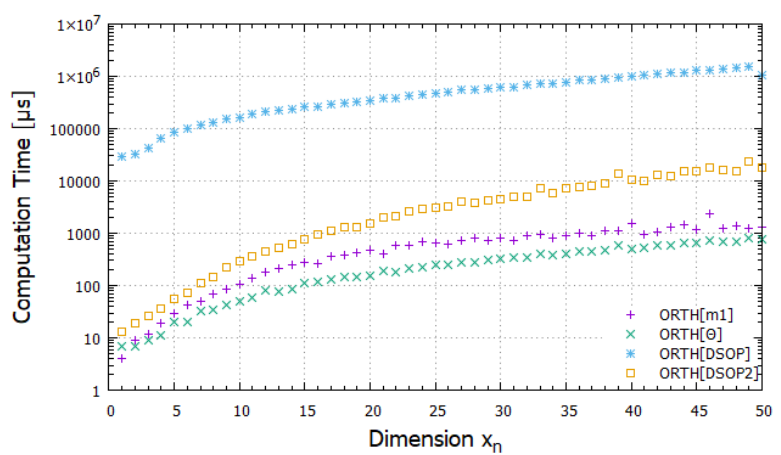

(c) SOPs with 10 product terms

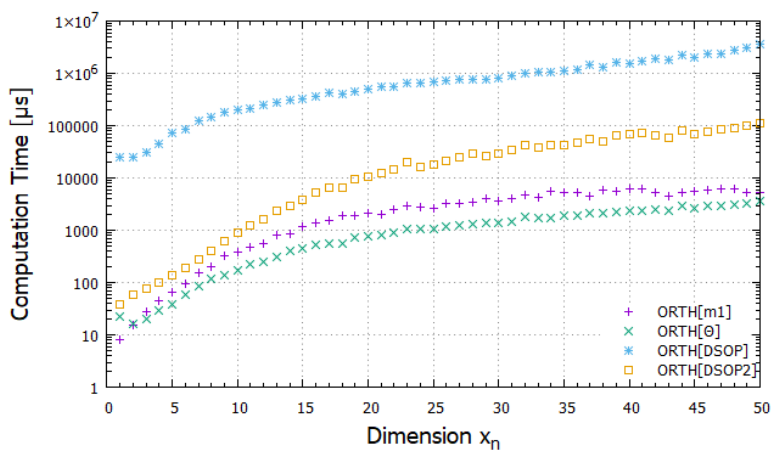

(e) SOPs with 20 product terms

Figure 6: Comparison in computation time

tic logic minimizer. In contrast to our function $a b s o r b()$ the number of terms in the minimized result is fewer by "espresso.exe". This step of minimization is important because a further calculation of a dSOP with fewer number of product terms needs fewer operations and is carried out by reduced computation time. In this case, a further calculation of a dSOP such as the Boolean Differential Calculus (BDC) is performed with fewer number of product terms and thus reduces the number of further operations and the

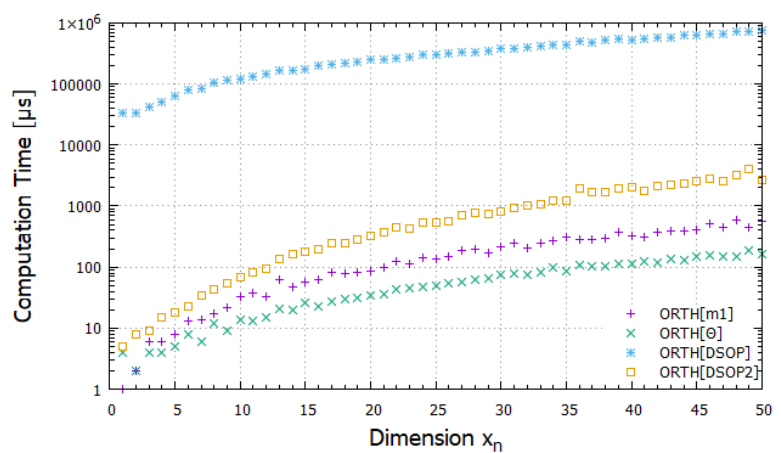

(b) SOPs with 5 product terms

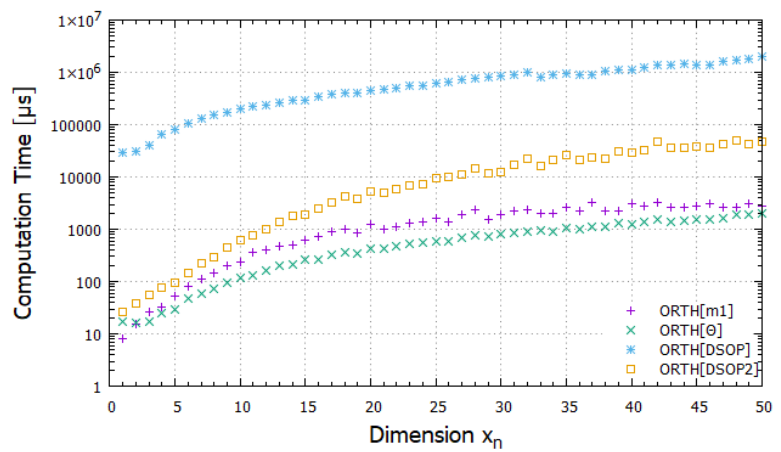

(d) SOPs with 15 product terms

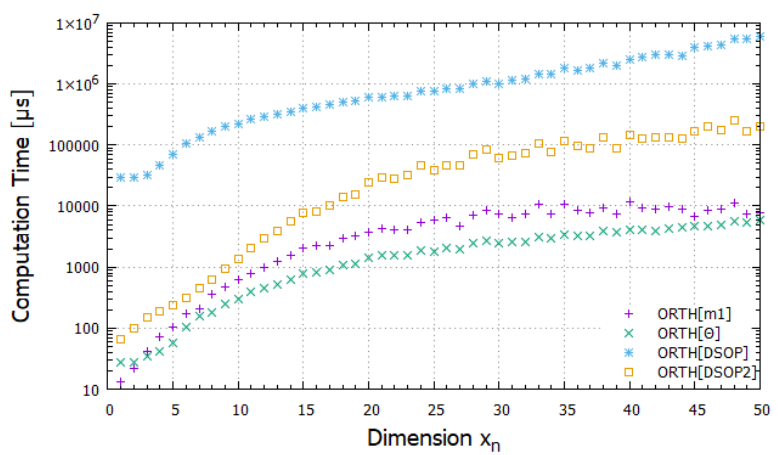

(f) SOPs with 25 product terms

number of calculation steps which will certainly affect the computation time.

\subsection{Comparison in Computation Time}

In this section the comparison of all four approaches relating to the computation time in respect to $N=$ $\{2,5,10,15,20,25\}$ and $x_{n}=\{1,2, \ldots, 50\}$ as shown in the Figures 6a) - f) is given. The corresponding average values of the calculation times and the ratios to 
Table 4: Average of the computation times and relation to $O R T H[\ominus]$

\begin{tabular}{|c|c|c|c|c|c|c|c|}
\hline$x_{n}$ & $\begin{array}{r}\mathrm{ORTH}[\ominus] \\
\text { in } \mu \mathrm{S}\end{array}$ & $\begin{array}{r}\text { ORTH[DSOP] } \\
\text { in } \mu \mathrm{S}\end{array}$ & $\begin{array}{r}\text { ORTH[DSOP2] } \\
\text { in } \mu \mathrm{S}\end{array}$ & $\begin{array}{r}\mathrm{ORTH}[\mathrm{m} 1] \\
\text { in } \mu \mathrm{S}\end{array}$ & 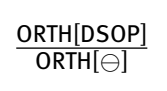 & 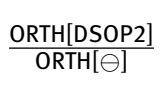 & $\frac{\mathrm{ORTH}[\mathrm{m} 1]}{\mathrm{ORTH}[\ominus]}$ \\
\hline 2 & 7,02 & 196545,63 & 100,48 & 33,09 & 27996,18 & 14,31 & $\overline{4,71}$ \\
\hline 5 & 65,79 & 338287,65 & 988,83 & 191,86 & 5142,02 & 15,03 & 2,92 \\
\hline 10 & 297,52 & 580726,60 & 5586,03 & 667,70 & 1951,86 & 18,78 & 2,24 \\
\hline 15 & 720,68 & 714265,14 & 15323,50 & 1520,70 & 991,10 & 21,26 & 2,11 \\
\hline 20 & 1298,12 & 948014,46 & 31221,00 & 3023,73 & 730,30 & 24,05 & 2,33 \\
\hline 25 & 2211,61 & 1443192,99 & 65237,83 & 5149,57 & 652,55 & 29,50 & 2,33 \\
\hline$\varnothing$ & 766,79 & 703505,41 & 19742,95 & 1764,44 & 917,47 & 25,75 & 2,30 \\
\hline
\end{tabular}

the method $O R T H[\ominus]$ are given in the Table 4. The computation times of method $O R T H[\ominus]$ is faster in comparison to the heuristic ORTH[DSOP], ORTH[m1] and the varied form $O R T H[D S O P 2]$. The complexity class of $O R T H[\ominus]$ totals up to $\Theta\left(n^{5}\right)$. The distinction is that the noval method $O R T H[\ominus]$ calculates the orthogonalizing difference-building $\ominus$ consistently no matter if two product terms are orthogonal or not. As this consideration takes place in method $O R T H[\mathrm{~m} 1]$ in [1], the computation time is likely to be deteriorated. Thus unnecessary calcu-

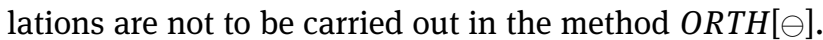
By the use of "espresso.exe" in ORTH[DSOP] the procedure time of orthogonalization is more slowly. This is also confirmed by replacing the function with absorb(), which is shown by the charts of ORTH[DSOP2]. Therefore, its computation time is decelerated. Due to the replacement of the minimization function by $\operatorname{absorb}()$ in $O R T H[D S O P 2]$ the calculation time gets faster in comparison to $O R T H[D S O P]$. However, it is still higher than the computation time of the novel method $O R T H[\ominus]$. In summary, it has to be clarified here that the new method has faster computation time than the other approaches. $O R T H[\ominus]$ is approximately 1000 times faster in comparison to $O R T H[D S O P]$, approximately 25 times faster than $O R T H[D S O P 2]$ and twice as fast than $O R T H[m 1]$. Even if the two sub-functions $\operatorname{absorb}()$ and $\operatorname{sort}()$ are excluded, the method $O R T H[\ominus]$ provides computation time, which are reduced, as shown in Figure 6a) - f). The measurements are limited to the dimension $x_{n}=50$. Against this, for dimension $x_{n}>50$ similar results are expected.

\section{Summary and Conclusions}

This work introduced a generally valid method of »orthogonalizing difference-building $\ominus$ « which is used to calculate the orthogonal difference of two product terms. Fur- thermore, rules for this method were explained which must be followed to get correct results. By a novel formula based on the combining technique $\ominus$ every Sum of Products (SOP) can easily be orthogonalized mathematically. Thus, we get disjoint Sum of Products (dSOP). A minimized dSOP can also be reached by two additional procedures of sorting and absorbing of terms before the process of orthogonalization. By sorting the product terms of a SOP are resorted from smaller number of variables to higher number of variables. This resorting brings an advantage of approximately $17 \%$ and $26 \%$ depending on $N$ to reach minimized dSOP. The corresponding Algorithm $O R T H[\ominus]$ was compared to other algorithms $O R T H[D S O P], O R T H[D S O P 2]$ and ORTH[m1] in their number of product terms in the calculated dSOP and the computation time. ORTH[DSOP] determines fewer number of product terms in contrast to $O R T H[\ominus]$. However, the reduction of the product terms by $O R T H[\ominus]$ is about $50 \%$ in contrast to $O R T H[m 1]$. Furthermore, the novel method $O R T H[\ominus]$ provides approximately 1000 times faster computation in comparison to $O R T H[D S O P]$ and is approximately 25 times faster in comparison to $O R T H[m 1]$. The number of terms in the orthogonalized result by the method $O R T H[\ominus]$ can probably reduced by an additional absorption of the disjoint product terms. For that, a postfunction for absorption could be developed, which retains the property of orthogonality.

\section{References}

[1] D. Bochmann, Binäre Systeme - Ein Boolean Buch. Hagen, Germany: LiLoLe-Verlag, 2006.

[2] B. Steinbach and C. Posthoff, "An extended theory of boolean normal forms," in Proc. 6th Annual Hawaii International Conference on Statistics, Mathematics and Related Fields, (Hawaii, USA), pp. 1124-1139, 2007.

[3] Y. Can, Neue Boolesche Operative Orthogonalisierende Methoden und Gleichungen. Erlangen, Germany: FAU University Press, 
1 ed., 2016.

[4] Y. Crama and P. Hammer, Boolean Functions - Theory, Algorithms, and Applications. Cambridge, UK: Cambridge University Press, 2011.

[5] A. Bernasconi, V. Ciriani, F. Luccio, and L. Pagli, "New Heuristic for DSOP Minimization," in Proc. 8th International Workshop on Boolean Problems (IWSBP), (Freiberg (Sachsen), Germany), 2008.

[6] H. J. Zander, Logischer Entwurf binärer Systeme. Berlin, DDR: Verlag Technik, 1989.

[7] B. Steinbach, "The Boolean Differential Calculus - Introduction and Examples," in Proc. Reed-Muller Workshop 2009, (Naha, Okinawa, Japan), pp. 107-117, 2009.

[8] Y. Can, H. Kassim, and G. Fischer, "New Boolean Equation for Orthogonalizing of Disjunctive Normal Form based on the Method of Orthogonalizing Difference-Building," Journal of Electronic Testing. Theory and Applicaton (JETTA), vol. 32, no. 2, pp. 197208, 2016.
[9] Y. Can, H. Kassim, and G. Fischer, "Orthogonalization of DNF in TVL-Arithmetic," in Proc. 12th International Workshop on Boolean Problems (IWSBP), (Freiberg (Sachsen), Germany), 2016.

[10] C. Dorotska and B. Steinbach, "Orthogonal Block Change \& Block Building Using Ordered Lists of Ternary Vectors," in Proc. 5th International Workshop on Boolean Problems (IWSBP), (Freiberg (Sachsen), Germany), pp. 91-102, 2002.

[11] C. Posthoff and B. Steinbach, Logikentwurf mit XBOOLE. Algorithmen und Programme. Berlin, Germany: Verlag Technik $\mathrm{GmbH}, 1991$.

[12] C. Posthoff and B. Steinbach, Binäre Gleichungen - Algorithmen und Programme. Karl-Marx-Stadt (Chemnitz), DDR: Technische Universität Karl-Marx-Stadt, 1979. 